\title{
Effective Capacity Analysis of Reconfigurable Intelligent Surfaces Aided NOMA Network
}

\section{Geng Li}

Henan Polytechnic University

\section{Huiling Liu}

Henan Polytechnic University

\section{Gaojian Huang}

Henan Polytechnic University https://orcid.org/0000-0002-0450-990X

\section{Xingwang Li ( $\sim$ lixingwangbupt@gmail.com )}

Henan Polytechnic University

\section{Bichu Raj}

American College of the Middle East

\section{Ferdi Kara}

Zonguldak Bulent Ecevit Universitesi

\section{Research Article}

Keywords: effective capacity, non-orthogonal multiple access, reconfigurable intelligent surfaces

Posted Date: September 7th, 2021

DOl: https://doi.org/10.21203/rs.3.rs-829162/v1

License: (c) (i) This work is licensed under a Creative Commons Attribution 4.0 International License. Read Full License

Version of Record: A version of this preprint was published at EURASIP Journal on Wireless Communications and Networking on December 1st, 2021. See the published version at https://doi.org/10.1186/s13638-021-02070-7. 


\title{
RESEARCH
}

\section{Effective Capacity Analysis of Reconfigurable Intelligent Surfaces Aided NOMA network}

\author{
Geng $\mathrm{Li}^{1}$, Huiling $\mathrm{Liu}^{1}$, Gaojian Huang ${ }^{1}$, Xingwang $\mathrm{Li}^{1}$, Bichu Raj ${ }^{2}$ and Ferdi Kara ${ }^{3}$
}

${ }^{*}$ Correspondence:
gaojianhuang@yahoo.com
${ }^{1}$ Physics and Electronic
Information Engineering, Henan
Polytechnic University, Jiaozuo
454003 , China
Full list of author information is
available at the end of the article

\begin{abstract}
The future sixth generation $(6 \mathrm{G})$ is going to face the significant challenges of massive connections and green communication. Recently, reconfigurable intelligent surfaces (RIS) and non-orthogonal multiple access (NOMA) have been proposed as two key technologies to address the above problems. Motivated by this fact, we consider a downlink RIS-aided NOMA system, where the base station seeks to communicate with two NOMA users with the aid of a RIS. Considering future network supporting real-time service, we investigate the system performance with the view of effective capacity (EC), which is an important evaluation metric of sensitive to delay sensitive system. Based on this basis, we derive the analytical expressions of the EC of the near and far users. To obtain more useful insights, we deduce the analytical approximation expressions of the EC in the low signal-to-noise-ratio (SNR) approximation by utilizing Taylor expansion. In order to compare, we provide the results of orthogonal multiple access (OMA). It is found that 1 ) The number of RIS components and the transmission power of the base station have important effects on the performance of the considered system model. 2) Compared with OMA, NOMA system has higher effective capacity due to the short transmission time.
\end{abstract}

Keywords: effective capacity; non-orthogonal multiple access; reconfigurable intelligent surfaces

\section{Introduction}

For the past few years, the sixth generation (6G) mobile communication technology has attracted a lot of attentions because of its high transmission rate, high reliability, high capacity and other advantages. Thus it has great practical and economic value for the industry and Internet of Things (IoT) $[1,2,3,4]$. The application scenario of the IoT is an integration of a variety of emerging technologies, which has become the most popular research at present. The rise of the IoT has put forward higher requirements for communication technology. On the other hand, for the future wireless networks, large-scale access, high energy consumption and hardware costs are the main characteristic for the future wireless networks. Therefore, it is imperative to find solutions with low power consumption and high economic benefits to meet users' high requirements for quality of service (QoS) and data rate $[5,6]$. Fortunately, the concept of Backscatter and reconfigurable intelligent surfaces (RIS) have been introduced into wireless communication research [7, 8, 9]. Both of these are passive devices that no longer use an active transmitter but instead reflect surrounding radio frequency (RF) signals as a passive array, thus not resulting in extra power consumption and no need for specialized energy supply [10]. The 
difference between Backscatter and RIS is that the signal reflected by RIS is not processed. On the contrary, signals reflected by backscatter devices are processed, may resulting in processing delays and complexity. So, RIS performs better in solving the problem of energy consumption and hardware cost reduction in wireless communication systems.

As a new paradigm, RIS is equipped with multiple reflective elements composed of two-dimensional artificial electromagnetic materials. Each reflective element is controlled by the software, then the reflected phase and angle of the incident RF signal can be flexibly adjusted. By adjusting RIS, the ideal effect is obtained and the performance of wireless communication system is improved [11]. RIS can greatly increase the channel capacity, and can be flexibly configured on walls, ceilings, and outer surfaces of buildings. It has extremely low power consumption and is environmentally friendly, which can availably improve the energy efficiency of the communication system [12]. Compared with the existing technologies, RIS can achieve deterministic and programmable control of wireless environment behavior. Based on these advantages, a great deal of researchers have focused on the research works of these aspects of RIS. In [13], the authors developed an optimization of joint the transmit power allocation and the phases shifts of the surface reflecting elements of each link to maximize energy efficient of downlink RIS-assisted multiuser communication systems. The author solved the issue of minimizing the total transmission power in the RIS wireless system in [14]. For the performance analysis aspects, Jung et al. analyzed the reliability of the RIS system and derived the expressions of the RIS system's uplink sum rate as well as outage probability [15]. The authors in [16] analyzed the capacity degradation problem of RIS network with hardware damage. In addition, some researchers also studied RIS in combination with other technologies. In [17], the channel estimation of RIS-aided millimeter-wave (mmWave) multiple-input multiple-output (MIMO) system was estimated through adopting a two-stage iterative reweighted method to find the perfect channel state information. In [18], authors proposed an adaptive phase shifter design of RIS -aided mmWave MIMO system based on the hierarchical codebooks and mobile station feedback. The author of [19] introduced RIS to simultaneous wireless information and power transfer (SWIPT) and used RIS to enhance energy harvesting. Zhiguo Ding proposed a simple design of RIS-assisted non-orthogonal multiple access (NOMA) downlink transmission, which can improve the spectral efficiency and connectivity in [20]. All these studies show that the combination of RIS and other technologies is very promising.

RIS can reconstruct the wireless environment, which promotes researchers to introduce RIS into NOMA system. NOMA is a promising technology for 5G and has attracted wide attention in academia and industry [21, 22, 23, 24, 25]. NOMA is considered to have obvious advantages in solving problems of spectrum scarce and devices access, and is an effective method to solve large-scale access [26, 27]. The pivotal idea of NOMA is that multiple users at the transmitter and receiver use the identical time/frequency domain resources, and the transmitter directly sends the superposition code of multiple users. For ensuring fairness, the allocation power to users are inversely proportional with channel gain. That is, users with large channel gain allocate more power, vice versa. At the receivers, the information can 
be recovered by using serial interference cancellation (SIC) [28]. Therefore, NOMA technology has been widely recognized as a method to improve the quality of mobile network access, widely used in a variety of communication scenarios, and has a promising prospect $[29,30]$. However, the NOMA technology is not without its drawbacks. The realization of NOMA technology requires high-cost multiple input multiple output MIMO. When the wireless system has numerous users, it is difficult to achieve better performance by relying solely on NOMA technology. To this end, RIS provides a new idea for improving the performance of the NOMA system. Many studies have also shown that RIS can effectively improve the NOMA system's performance. In [31], the performance of NOMA and OMA in the RIS-assisted downlink communication network was compared, and a low-complexity algorithm with close to optimal performance was proposed. Reference [32] studied coherent phase shifting and random discrete phase shifting on the impact of the RIS-aided NOMA system. The authors of [33] studied the RIS-aided NOMA system's physical layer security performance. The outage probability of the RIS-aided NOMA system with hardware damage is analyzed in [34]. Further, in [35], a RIS-aided NOMA system has a single eavesdropper with residual hardware impairment was proposed, and the performance of physical layer security is studied.

Whereas, the current researches on the RIS-aided NOMA communication system mainly focuses on the analysis of channel capacity, outage probability, etc., and have not considered the influence of delay on system performance. Thus it is impossible to make an accurate analysis of the communication real-time business requirements. For purpose of analyzing the influence of delay on the communication system, Wu et al. put forward the concept of effective capacity (EC) in [36], which is used to quantify the accessibility of various wireless systems under the limit of the delay QoS. EC can provide a key point to characterize the impact of statistical delay QoS on system performance. In addtion, in [37] a unified pattern for analyzing effective rate in wireless communication system based on multi-input single-output (MISO) arbitrary correlated generalized $\mathrm{K}$ fading channels and hyper Fox's $\mathrm{H}$ fading channels was proposed. To reduce complexity and energy consumption, a transmit antenna selection (TAS) schemed was designed, and the closed form expression of MISO TAS system was derived in [38]. This was further extended to generalized fading channels in [39], where in the approximate EC of MISO systems with $\kappa-\mu$ fading channels by using momnet matching method was investigated.

As far as we know, there are not relevant works on studying the EC of the RISaided NOMA communication system. From this motivation, in this paper, we propose a wireless communication system model with RIS-aided NOMA technology with two users. The main contributions are summarized as follows:

- We consider a downlink RIS-aided NOMA system. The base station sends information to two users which are in NOMA cases with the aid of RIS with $\mathrm{N}$ reflection elements. Considering delay-sensitive requirements, we introduce a key performance evaluation metric of EC into the considered system.

- We first derive the approximate expressions for the EC of the near and far users. The EC of RIS-aided NOMA system for the near user relies on transmission power, power allocation coefficients $\xi_{n}, \xi_{f}$, number of RIS, QoS exponent, bandwith B. While the EC for the far user only determined by bandwith B and power allocation factors $\xi_{n}, \xi_{f}$. 
- We deduce the asymptotic behavior of EC in the low signal-to-noise-ratio (SNR) approximation by invoking Taylor expansion. Moreover, in order to comparison, we provide the results of orthogonal multiple access (OMA).

The rest of this paper's structure is as follows. The system model of this paper and the concept of the EC are introduced in Section 2. In section 3, we derive the analytical expression for the EC of single-input single-output (SISO) systems as well as the asymptotic analysis in the low SNR area. The simulation results and the summary of this paper are given in Section 4.

The main notations used in this paper are as follows. In this paper, we use capital boldface to indicate a matrix and lowercase boldface to indicate a vector. We use $\mathrm{E}\{\cdot\}$ for the expectation operation. Bessel functions of the second kind are denoted by $K_{v}(\cdot)$. The conjugate transpose of a matrix or vector are denoted by the notations $(\cdot)^{H} \cdot \mathcal{C N} \sim\left(\mu, \sigma^{2}\right)$ denotes Gaussian which has the mean of $\mu$ and the variance of $\sigma^{2}$. The probability density function (PDF) of a random variable $X$ is expressed by $f_{X}(\cdot)$.

\section{Methods}

\subsection{System model}

We consider a RIS-aided NOMA network as illustrated in Fig. 1. It includes a base station (BS), a RIS consists with $\mathrm{N}$ reflection elements, a far user (FU) and a near user (NU). There is no direct connection between the BS and the users due to the non-line-of-sight. We assume that: i) all nodes are single antenna; ii) all channels obey independent Rayleigh fading. It is noted that although the Rayleigh channel is not fully applicable in the physical model of RIS, for the sake of simple analysis, the Rayleigh channel is adopted in this paper, and other fading channels will be studied in our future research. We denote $\mathbf{h}_{s r}=\left[h_{s r}^{1} \ldots h_{s r}^{n} \ldots h_{s r}^{N}\right]$, where $\mathbf{h}_{s r} \sim\left(0, \Omega_{s r}\right)$ as the baseband equivalent fading channels between BS and RIS. $\mathbf{h}_{r n}=\left[h_{r n}^{1} \ldots h_{r n}^{n} \ldots h_{r n}^{N}\right]$ and $\mathbf{h}_{r f}=\left[h_{r f}^{1} \ldots h_{r f}^{n} \ldots h_{r f}^{N}\right]$ denote channels from the RIS to NU and FU, respectively. Where $\mathbf{h}_{r n} \sim\left(0, \Omega_{r n}\right)$ and $\mathbf{h}_{r f} \sim\left(0, \Omega_{r f}\right)$.

Figure 1 System Model

First, the BS sends superposition signals $x_{n}, x_{f}$ to UN and UF. The total transmit power budget of the BS is $P_{s}$. The power allocation coefficients of NU and FU are $\xi_{n}$ and $\xi_{f}$, which satisfy with $\xi_{n}<\xi_{f}, \xi_{n}+\xi_{f}=1$. Following principle of RIS, the received signals at users are given as

$$
\begin{aligned}
& y_{n}=\mathbf{h}_{s r}^{H} \boldsymbol{\Theta} \mathbf{h}_{r n}\left(\sqrt{\xi_{n} P_{s}} x_{n}+\sqrt{\xi_{f} P_{s}} x_{f}\right)+n_{n}, \\
& y_{f}=\mathbf{h}_{s r}^{H} \boldsymbol{\Theta} \mathbf{h}_{r f}\left(\sqrt{\xi_{n} P_{s}} x_{n}+\sqrt{\xi_{f} P_{s}} x_{f}\right)+n_{f} .
\end{aligned}
$$

where $\boldsymbol{\Theta}=\operatorname{diag}\left(\beta e^{j \theta_{1}}, \ldots, \beta e^{j \theta_{n}}, \ldots, \beta e^{j \theta_{N}}\right)$ is a diagonal matrix, $\beta \in[0,1]$ denotes the fixed reflection amplitude coefficient and $\theta_{n} \in[0,2 \pi)$ denotes the phase shift of the n-th reflecting element of the IRS. $n_{n}$ and $n_{f}$ are the additive white Gaussian noise (AWGN) and satisfy $n_{n} \sim \mathcal{N}\left(0, \sigma_{n}^{2}\right)$ and $n_{f} \sim \mathcal{N}\left(0, \sigma_{f}^{2}\right)$. 
For NU, in the NOMA scene, interference from FU is eliminated due to the existence of SIC, then the SNR of UN can be given as

$$
\gamma_{n}=\frac{\xi_{n} P_{s}\left|\mathbf{h}_{s r}^{H} \boldsymbol{\Theta} \mathbf{h}_{r n}\right|^{2}}{\sigma^{2}},
$$

For FU, which is different from NU, it does not have SIC, we obtain SNR at FU as

$$
\gamma_{f}=\frac{\xi_{f} P_{s}\left|\mathbf{h}_{s r}^{H} \boldsymbol{\Theta} \mathbf{h}_{r f}\right|^{2}}{\sigma^{2}+\xi_{n} P_{s}\left|\mathbf{h}_{s r}^{H} \boldsymbol{\Theta} \mathbf{h}_{r f}\right|^{2}} .
$$

\subsection{Effective Capacity}

Effective capacity is the maximum constant arrival rate at the queue of the transmitter under the condition of satisfying statistical QoS constraints [34]. The mathematical expression is

$$
E C(\alpha)=-\lim _{t \rightarrow \infty} \frac{1}{\alpha t} \ln \left[E\left(e^{-\alpha s(t)}\right)\right]
$$

where $t$ is the block-length. $s(t)$ the cumulative service process. $\alpha$ is QoS exponent, which is related with both delay violation probability and length of a queue. When $\alpha$ approaches zero, it corresponds to delay tolerance communication, while when $\alpha$ approaches infinity, it turns to delay limited communication. It is given as

$$
\alpha=-\lim _{x \rightarrow \infty} \frac{\ln \operatorname{Pr}(L>x)}{x}
$$

where $L$ is the equilibrium queue-length of the buffer at the transmitter.

In this study, it is assumed that the BS has perfect channel state information (CSI), so service rate $s(t)$ at time slot $t$ can be represented by Shannon capacity $c(t)$. The mathematical expression of $c(t)$ can be given as

$$
c(t)=B \log _{2}(1+\gamma(t))
$$

where B is bandwidth.

We assume that independent and identical block fading, so Eq. (5) can be rewritten as

$$
E C(\alpha)=-\frac{1}{\alpha} \ln \left[E\left(e^{-\alpha c(t)}\right)\right]
$$

\section{Performance Analysis}

In this section, the approximate analytical closed-form expressions for the EC of NU and FU in high and low SNRs are derived. In the high SNR analysis, we use the dominant term to approximate SNR, and carry out the approximate analysis. While in the low SNR analysis, we deduce the asymptotic analysis by invoking the Taylor series. 


\subsection{Approximate Analysis}

In this subsection, we derive the EC closed-form expressions of NU and FU, which is obtained by taking SNR large.

Theorem 1 The closed-form expression at $N U$ is given by

$$
\begin{aligned}
E C_{n}(\alpha)= & \frac{B}{\ln (2)} \ln \left(\frac{\xi_{n} P_{s} \beta^{2}}{\sigma^{2}}\right)+\frac{2 B}{\ln (2)} \ln \left(\sigma_{a} \sigma_{b}\right)+\frac{1}{\alpha} \ln (\Gamma(N)) \\
& -\frac{1}{\alpha} \ln \left(\Gamma\left(-\frac{\alpha B}{\ln (2)}+N\right) \Gamma\left(-\frac{\alpha B}{\ln (2)}+1\right)\right) .
\end{aligned}
$$

where $\Gamma(\cdot)$ is the gamma function.

Proof See the Appendix 1.

Theorem 2 The closed-form expression at FU is given by

$$
E C_{f}(\alpha)=-\frac{B}{\ln (2)} \ln \left(\frac{\xi_{n}}{\xi_{f}}\right) .
$$

Proof See the Appendix 2.

Remark 1 Because the high SNR approximation is used in the calculation, from Theorem 1, Theorem 2, we can see that: 1) For NU, the EC is logarithmic to the transmit power $P_{s}$ and channel parameters $\sigma_{a} \sigma_{b}$; 2) The EC value of $F U$ is a constant in the high SNR.

\subsection{Low-SNR Approximate Analysis}

For the case of low SNR, a similar methodology to that in [39] is adopted. At NU and FU, the second-order Taylor expansion of SNR is used, $\gamma \rightarrow 0^{+}$

$$
E C(\alpha)=\ddot{E C}(\alpha) \gamma+\ddot{E C}(\alpha) \frac{\gamma^{2}}{2}+o\left(\gamma^{2}\right),
$$

where $\operatorname{EC}(\alpha)$ and $\ddot{\operatorname{EC}}(\alpha)$ denote the first- and second-order derivatives of the approximate $\mathrm{EC}$ with regard to the SNR $\gamma \rightarrow 0^{+}$respectively.

For NU, they can be shown that

$$
\begin{aligned}
& \dot{E C_{n}}(\alpha)=\frac{B \mathrm{E}(Q)}{\ln (2)}, \\
& \ddot{E C_{n}}(\alpha)=\frac{B}{\ln (2)}\left(\frac{-\alpha B}{\ln (2)}-1\right) \mathrm{E}\left(Q^{2}\right)+\frac{\alpha B^{2}}{\ln ^{2}(2)}(\mathrm{E}(Q))^{2},
\end{aligned}
$$

where $Q=\xi_{\mathrm{n}} \beta^{2}\left(\mathbf{a}^{T} \mathbf{b}\right)^{2}$, using Eq. (24), the means of $Q$ and $Q^{2}$ can be respectively given by

$$
\mathrm{E}(Q)=\frac{\xi_{n} \beta^{2} \Gamma(N+2) \Gamma(3)\left(\sigma_{a} \sigma_{b}\right)^{5}}{\Gamma(N)},
$$




$$
\mathrm{E}\left(Q^{2}\right)=\frac{\left(\xi_{n} \beta^{2}\right)^{2}\left(\sigma_{a} \sigma_{b}\right)^{4} \Gamma(N+2) \Gamma(3)}{\Gamma(N)} .
$$

Substitute Eq. (12) - (15) into Eq. (11), we obtain the EC expression of NU with low SNR approximation.

FU is similar to NU, they have the same ideas and calculations, we can get

$$
\begin{aligned}
& \dot{E C_{f}}(\alpha)=\frac{B \mathrm{E}(G)}{\ln (2)} \\
& \ddot{E C_{f}}(\alpha)=-\frac{B}{\ln (2)}\left(\frac{\alpha B}{\ln (2)}+1\right) \mathrm{E}\left(G^{2}\right)+\frac{2}{\alpha} \mathrm{E}(G F)-\frac{B}{\ln (2)}(\mathrm{E}(G))^{2},
\end{aligned}
$$

where $G=\xi_{f} \beta^{2}\left(\mathbf{a}^{T} \mathbf{c}\right)^{2}, F=\xi_{n} \beta^{2}\left(\mathbf{a}^{T} \mathbf{c}\right)^{2}$. The values of $G, G F$ and $G^{2}$ are respectively given by

$$
\begin{aligned}
& \mathrm{E}(G)=\frac{\xi_{f} \beta^{2} \Gamma(N+2) \Gamma(3)\left(\sigma_{a} \sigma_{c}\right)^{5}}{\Gamma(N)}, \\
& \mathrm{E}\left(G^{2}\right)=\frac{\left(\xi_{f} \beta^{2}\right)^{2}\left(\sigma_{a} \sigma_{c}\right)^{4} \Gamma(N+2) \Gamma(3)}{\Gamma(N)}, \\
& \mathrm{E}(G F)=\frac{\xi_{n} \xi_{f} \beta^{4}\left(\sigma_{a} \sigma_{c}\right)^{4} \Gamma(N+2) \Gamma(3)}{\Gamma(N)} .
\end{aligned}
$$

Substitute Eq. (16) - (20) into Eq. (11), we obtain the EC expression of FU with low SNR approximation.

Remark 2 It can be seen from the second-order Taylor expansion of low SNR that: 1) The EC is the sum of power functions related to the transmission SNR. 2) When the power allocation coefficient increases, the EC of NU increases.

\section{Results and Discussion}

In this section, we provide numerical analysis to verify the accuracy of the analysis results for the considered system model. In the case of a random channel, we implement the generation of Rayleigh random variables in $10^{5}$. We set the parameters as follows: bandwidth $B=1$, variance of system noise $\sigma^{2}=1$, reflection amplitude coefficient $\beta=1$. The purpose is to see the influence of the transmitted power and RIS elements on the EC of the system. For power allocation factor of NOMA, we set $\xi_{f}=0.9, \xi_{n}=0.1$.

Figure 2 The EC relation with SNR on NU and FU

As shown in Fig. 2, it illustrates the effect of SNR on the EC of NU and FU with the Monte Carlo results, respectively. The behavior of ECs become tight when SNR increases based on Eq. (9) and (10), the approximate result is obtained when SNR is infinity. The simulation results show that the EC of NU increases linearly with the increase of SNR. But as SNR value increases, the theoretical EC of FU increases 
slowly and converges to a ceiling because FU suffers from NU's interference when it detects its own signals. The approximation of EC is a constant as we analyzed in Remark 1. In addition, it is clearly to see the important influence of the number of RIS elements $N$ on the EC when applying $N=1,3,5$. That is the EC certainly increases as the number of RIS elements $N$ increases, which is consistent with the result of [40].

Figure 3 The EC relation with SNR in case of NOMA and OMA at NU

Figure 4 The EC relation with SNR in case of NOMA and OMA at NU

Figure 5 Comparison of the sum of EC between NOMA and OMA

Fig. 3 and 4 plot the relationship between ECs versus SNR in the case of NOMA and OMA for the NU and FU, respectively. We can observe that the EC of NU for RIS-aided NOMA is superior to RIS-aided OMA. However, for FU, the EC in OMA is better than that in NOMA. That is because FU suffers from NU's interference in NOMA. Hence, we conclude that RIS-aided NOMA can significantly improve the EC of NU. Moreover, to better analyze the EC of the whole system, the sum EC of RIS-aided NOMA and OMA are compared in Fig. 5 with different number of RIS elements. The simulations show that for the whole system, the EC is in the lead when applies NOMA. Thus it can be seen the sum EC is dominated by NU. Another observation is that the increase of the number of RIS elements $N$ has a positive effect on the sum EC of the system.

Figure 6 EC approximation with low-SNR with $N=3$

To exhibit the impact of low SNR on the EC in Eq. (11), the approximate behavior of the EC in the case of low SNR is shown in Fig. 6. We take the SNR from $-25 d B$ to $-10 d B$ and the number of RIS elements $N=3$ as an example. The low SNR approximation of NU, FU and the sum EC of the system are compared. The good match between the approximation and the simulation in lower SNR condition confirms the derived expressions are right. Besides, the result shows that the EC increases exponentially with SNR in the condition of low SNR regions.

\section{Conclusion}

In this paper, we considered a downlink wireless communication system with RISaided NOMA technology. To consider delay-sensitive requirements, the EC as the evaluation metric was taken. First, we derived the approximate expressions for the EC of NU and FU. Through the simulation, the main factors affect the EC are the number of RIS, transmit power budget and QoS exponent, as well as power allocation coefficients and bandwith. In addition, we provided the relationship between 
ECs and SNR in the case of NOMA and OMA and concluded that the EC is excellent when applies NOMA for the whole system. Final, the asymptotic expression of EC by invoking Taylor expansion at low SNR is given, which offers a theoretical basis for the actual deployment of the system.

To sum up, the wireless communication system combined with RIS and NOMA has strong advantages and can meet the requirements of large-scale connection. The integration of RIS and NOMA with different fields will have greater development in the future.

\section{Appendix}

\section{Appendix 1 : Proof of Theorem 1}

Let's express $\mathbf{h}_{s r}, \mathbf{h}_{r n}$ and $\mathbf{h}_{r f}$ in the form of $\mathbf{h}_{s r}=\mathbf{a} e^{j \mu_{m}}, \mathbf{h}_{r n}=\mathbf{b} e^{j \nu_{m}}$ and $\mathbf{h}_{r f}=\mathbf{c} e^{j \lambda_{m}}$, where $\mathbf{a}=\left(a_{1}, \ldots, a_{n}, \ldots, a_{N}\right), \mathbf{b}=\left(b_{1}, \ldots, b_{n}, \ldots, b_{N}\right), \mathbf{c}=\left(c_{1}, \ldots, c_{n}, \ldots, c_{N}\right)$, and variances are $\sigma_{a}^{2}, \sigma_{b}^{2}$, and $\sigma_{c}^{2}$, respectively. In this paper, we assume there are no phase shift errors, that is to say: $\theta_{m}=-\mu_{m}-\nu_{m}$ and $\theta_{m}=-\mu_{m}-\lambda_{m}$. So we can rewrite the SNR of UN is rewritten as

$$
\gamma_{n}=\frac{\xi_{n} P_{s} \beta^{2}\left|\mathbf{a}^{T} \mathbf{b}\right|^{2}}{\sigma^{2}}
$$

Based on the definition of the EC in Eq. (8), we can write the expression of the EC at NU as

$$
E C_{n}(\alpha)=-\frac{1}{\alpha} \ln \left[E\left(e^{-\alpha B \log _{2}\left(1+\gamma_{\mathrm{n}}\right)}\right)\right]
$$

By applying [41], let $U \triangleq \mathbf{a}^{T} \mathbf{b}$, we can give the PDF for $\mathrm{U}$ :

$$
f_{U}(u)=\frac{4 u^{N}}{\Gamma(N)\left(\sigma_{a} \sigma_{b}\right)^{N+1}} K_{N-1}\left(\frac{2 u}{\sigma_{a} \sigma_{b}}\right)
$$

According to probability theory, we can get the PDF of $X=U^{2}$

$$
f_{X}(x)=\frac{2 x^{\frac{N-1}{2}} K_{N-1}\left(\frac{2 x^{\frac{1}{2}}}{\sigma_{a} \sigma_{b}}\right)}{\Gamma(N)\left(\sigma_{a} \sigma_{b}\right)^{N+1}}
$$

According to the definition of expectation in probability knowledge, $E\left(e^{-\alpha B \log _{2}\left(1+\gamma_{n}\right)}\right)$ is written as

$$
E\left(e^{-\alpha B \log _{2}\left(1+\gamma_{\mathbf{n}}\right)}\right)=\int_{0}^{\infty} \mathrm{e}^{-\alpha B \log _{2}(1+\delta \mathrm{x})} f(x) d x,
$$

where $\delta=\frac{\xi_{n} P_{s} \beta^{2}}{\sigma^{2}}$. Then we replace Eq. (24) to Eq. (25), we have

$$
E\left(e^{-\alpha B \log _{2}\left(1+\gamma_{\mathbf{n}}\right)}\right)=\int_{0}^{\infty} \mathrm{e}^{-\alpha B \log _{2}(1+\delta x)} \frac{2 x^{\frac{N-1}{2}} K_{N-1}\left(\frac{2 x^{\frac{1}{2}}}{\sigma_{a} \sigma_{b}}\right)}{\Gamma(N)\left(\sigma_{a} \sigma_{b}\right)^{N+1}} d x
$$

In fact, $\delta$ term is a variate which is quite greater than $1, x$ is a number from zero to infinity. When $\delta \rightarrow \infty$, we have the following approximation $1+\delta \mathrm{x} \approx \delta \mathrm{x}$, based on the above discussion, Eq. (26) is written as

$$
E\left(e^{-\alpha B \log _{2}\left(1+\gamma_{\mathrm{n}}\right)}\right)=\frac{2}{\Gamma(N)\left(\sigma_{a} \sigma_{b}\right)^{N+1}} \int_{0}^{\infty} \mathrm{e}^{-\alpha B \log _{2}(\delta x)} x^{\frac{N-1}{2}} K_{N-1}\left(\frac{2 x^{\frac{1}{2}}}{\sigma_{a} \sigma_{b}}\right) d x
$$


We can rewrite $\mathrm{e}^{-\alpha B \log _{2}(\delta \mathrm{x})}$ as $(\delta \mathrm{x})^{-\frac{\alpha B}{\ln (2)}}$ by the exponential algorithm. Now, using [42, Eq. (6.561.16)], Eq. (27) is written as

$$
E\left(e^{-\alpha B \log _{2}\left(1+\gamma_{\mathrm{n}}\right)}\right)=\frac{\Gamma\left(-\frac{\alpha B}{\ln (2)}+N\right) \Gamma\left(-\frac{\alpha B}{\ln (2)}+1\right)}{\delta^{\frac{\alpha B}{\ln (2)}}\left(\sigma_{a} \sigma_{b}\right)^{\frac{2 \alpha B}{\ln (2)}} \Gamma(N)} .
$$

Finally, substituting Eq. (28) for Eq. (22), we can get the EC of NU, as is showed in Eq. (9). So, the proof is completed.

Appendix 2 : Proof of Theorem 2 Similar to NU, the SNR of FU as

$$
\gamma_{f}=\frac{\xi_{f} P_{s} \beta^{2}\left|\mathbf{a}^{T} \mathbf{c}\right|^{2}}{\sigma^{2}+\xi_{n} P_{s} \beta^{2}\left|\mathbf{a}^{T} \mathbf{c}\right|^{2}}
$$

The expression of the EC of $\mathrm{FU}$ as

$$
E C_{f}(\alpha)=-\frac{1}{\alpha} \ln \left[E\left(e^{-\alpha B \log _{2}\left(1+\gamma_{\mathrm{f}}\right)}\right)\right],
$$

The same, we can get the PDF of $V=\left|\mathbf{a}^{T} \mathbf{c}\right|^{2}$

$$
f_{V}(v)=\frac{2 v^{\frac{N-1}{2}} K_{N-1}\left(\frac{2 v^{\frac{1}{2}}}{\sigma_{a} \sigma_{c}}\right)}{\Gamma(N)\left(\sigma_{a} \sigma_{c}\right)^{N+1}}
$$

Then, $E\left(e^{-\alpha B \log _{2}\left(1+\gamma_{\mathrm{f}}\right)}\right)$ is written as

$E\left(e^{-\alpha B \log _{2}\left(1+\gamma_{\mathrm{f}}\right)}\right)=\frac{2}{\Gamma(N)\left(\sigma_{a} \sigma_{c}\right)^{N+1}} \int_{0}^{\infty}\left(\frac{\xi_{f} P_{s} \beta^{2} y}{\sigma^{2}+\xi_{n} P_{s} \beta^{2} y}\right)^{-\frac{\alpha B}{\ln (2)}} y^{\frac{N-1}{2}} K_{N-1}\left(\frac{2 y^{\frac{1}{2}}}{\sigma_{a} \sigma_{c}}\right) d y$

For $\left(\frac{\xi_{f} P_{s} \beta^{2} y}{\sigma^{2}+P_{s} \beta^{2} \xi_{n} y}\right)^{-\frac{\alpha B}{\ln (2)}}, \mathrm{y}$ is a number from zero to infinity, when $\mathrm{y} \rightarrow \infty$, we have the following

approximation $\left(\frac{\xi_{f} P_{s} \beta^{2} y}{\sigma^{2}+P_{s} \beta^{2} \xi_{n} y}\right)^{-\frac{\alpha B}{\ln (2)}} \approx\left(\frac{\xi_{f} y}{\xi_{n} y}\right)^{-\frac{\alpha B}{\ln (2)}}$. And then use the same calculation as NU, we can get the EC at FU, as is showed in Eq. (10) finally. The proof is completed.

\section{Acknowledgements}

Not applicable

\section{Funding}

This work was support in party by Henan Scientific and Technological Research Project under grant 212102210557, in part by Key Scientific Research Projects of Higher Education Institutions in Henan Province under grant 20A510007, and in part by the National Natural Science Foundation of China under grant 61601414.

\section{Abbreviations}

6G: The sixth generation; loT: Internet of things; Qos: Quality of service; RIS: Reconfigurable intelligent surfaces; RF: Radio frequency; mmWave: millimeter-wave; MIMO: Multiple-input multiple-output; SWIPT: Simultaneous wireless information and power transfer; NOMA: Non-orthogonal multiple access; SIC: Serial interference cancellation; EC: effective capacity; MISO: Multi-input single-output; SNR: Signal to noise ratio; OMA: Orthogonal multiple access; SISO: Single-input single-output; PDF: Probability density function; BS: base station; NU: Near user; FU: Far user; AWGN: Additive white Gaussian noise; CSI: Channel state information.

Availability of data and materials

The datasets used and/or analysed during the current study are available from the corresponding author on reasonable request.

Ethics approval and consent to participate Not applicable.

Competing interests

The authors declare that they have no competing interests. 
Consent for publication

Not applicable

\section{Authors' contributions}

Geng Li, Gaojian Huang and Xingwang Li propose the innovation ideals. Huiling Liu and Xingwang Li carry out the experiments. Huiling Liu carries out the original draft. Geng Li, Huiling Liu, Gaojian Huang, Xingwang Li, Bichu Raj and Ferdi Kara review the manuscript. All authors read and approved the final manuscript.

\section{Author details}

${ }^{1}$ Physics and Electronic Information Engineering, Henan Polytechnic University, Jiaozuo 454003, China. ${ }^{2}$ Industrial Engineering Technology Program, American College of the Middle East, 15453 Dasman, Kuwait. ${ }^{3}$ Wireless Communication Technologies Laboratory (WCTLab), Zonguldak Bulent Ecevit University, Zonguldak, 67100 Turkey.

References

1. Stoica, R.-A., De Abreu, G.T.F., Hara, T., Ishibashi, K.: Massively concurrent non-orthogonal multiple access for $5 \mathrm{G}$ networks and beyond. IEEE Access 7, 82080-82100 (2019)

2. Saad, W., Bennis, M., Chen, M.: A vision of $6 \mathrm{G}$ wireless systems: Applications, trends, technologies, and open research problems. IEEE Network 34, 134-142 (2020)

3. Pham, Q.-V., Fang, F., Ha, V.N., Piran, M.J., Le, M., Le, L.B., Hwang, W.-J., Ding, Z.: A survey of multi-access edge computing in $5 \mathrm{G}$ and beyond: Fundamentals, technology integration, and state-of-the-art. IEEE Access 8, 116974-117017 (2020)

4. Benjebbour, A., Saito, Y., Kishiyama, Y., Li, A., Harada, A., Nakamura, T.: Concept and practical considerations of non-orthogonal multiple access (NOMA) for future radio access. In: International Symposium on Intelligent Signal Processing and Communication Systems: 12-15 Nov. 2013; Okinawa, pp. 770-774 (2013)

5. Xu, Y., Gui, G., Gacanin, H., Adachi, F.: A survey on resource allocation for $5 \mathrm{G}$ heterogeneous networks: Current research, future trends, and challenges. IEEE Communications Surveys Tutorials 23, 668-695 (2021)

6. Guo, Y., Zhao, Z., He, K., Lai, S., Xia, J., Fan, L.: Efficient and flexible management for industrial internet of things: A federated learning approach. COMPUTER NETWORKS 192 (2021)

7. Yao, C., Liu, Y., Wei, X., Wang, G., Gao, F.: Backscatter technologies and the future of internet of things: Challenges and opportunities. Intelligent and Converged Networks 1, 170-180 (2020)

8. Di Renzo, M., Zappone, A., Debbah, M., Alouini, M.-S., Yuen, C., de Rosny, J., Tretyakov, S.: Smart radio environments empowered by reconfigurable intelligent surfaces: How it works, state of research, and the road ahead. IEEE Journal on Selected Areas in Communications 58, 2450-2525 (2020)

9. $\mathrm{Wu}, \mathrm{Q}$., Zhang, R.: Towards smart and reconfigurable environment: Intelligent reflecting surface aided wireless networ. IEEE Communications Magazine 58, 106-112 (2020)

10. Gong, S., Lu, X., Hoang, D.T., Niyato, D., Shu, L., Kim, D.I., Liang, Y.-C.: Toward smart wireless communications via intelligent reflecting surfaces: A contemporary survey. IEEE Communications Surveys Tutorials 22, 2283-2314 (2020)

11. Bai, T., Pan, C., Deng, Y., Elkashlan, M., Nallanathan, A., Hanzo, L.: Latency minimization for intelligent reflecting surface aided mobile edge computing. IEEE Journal on Selected Areas in Communications 38, 2666-2682 (2020)

12. Liu, Y., Liu, X., Mu, X., Hou, T., Xu, J., Di Renzo, M., Al-Dhahir, N.: Reconfigurable intelligent surfaces: Principles and opportunities. IEEE Communications Surveys Tutorials, 1-1 (2021)

13. Huang, C., Zappone, A., Alexandropoulos, G.C., Debbah, M., Yuen, C.: Reconfigurable intelligent surfaces for energy efficiency in wireless communication. IEEE Transactions on Wireless Communications 18, 4157-4170 (2019)

14. $\mathrm{Wu}, \mathrm{Q}$., Zhang, R.: Intelligent reflecting surface enhanced wireless network via joint active and passive beamforming. IEEE Transactions on Wireless Communications 18, 5394-5409 (2019)

15. Jung, M., Saad, W., Jang, Y., Kong, G., Choi, S.: Reliability analysis of large intelligent surfaces (LISs): Rate distribution and outage probability. IEEE Wireless Communications Letters 8, 1662-1666 (2019)

16. Hu, S., Rusek, F., Edfors, O.: Capacity degradation with modeling hardware impairment in large intelligent surface. In: IEEE Global Communications Conference (GLOBECOM): 09-13 Dec. 2020; United Arab Emirates, pp. 1-6 (2018)

17. He, J., Leinonen, M., Wymeersch, H., Juntti, M.: Channel estimation for RIS-aided mmWave MIMO systems. In: GLOBECOM 2020 - 2020 IEEE Global Communications Conference: 7-11 Dec. 2020; Taiwan, pp. 1-6 (2020)

18. He, J., Wymeersch, H., Sanguanpuak, T., Silven, O., Juntti, M.: Adaptive beamforming design for mmWave RIS-aided joint localization and communication. In: IEEE Wireless Communications and Networking Conference Workshops (WCNCW): 6-10 Apr. 2020; Seoul, pp. 1-6 (2020)

19. Yang, Z., Zhang, Y.: Optimal SWIPT in RIS-aided MIMO networks. IEEE Access, 1-1 (2021)

20. Ding, Z., Vincent Poor, H.: A simple design of IRS-NOMA transmission. IEEE Communications Letters 24 1119-1123 (2020)

21. Saito, Y., Benjebbour, A., Kishiyama, Y., Nakamura, T.: System-level performance evaluation of downlink non-orthogonal multiple access (NOMA). In: IEEE 24th Annual International Symposium on Personal, Indoor, and Mobile Radio Communications (PIMRC): 12-15 Nov. 2013; London, pp. 611-615 (2013)

22. Do, D.-T., Nguyen, M.-S.V., Jameel, F., Jäntti, R., Ansari, I.S.: Performance evaluation of relay-aided CR-NOMA for beyond 5G communications. IEEE Access 8, 134838-134855 (2020)

23. Do, D.-T., Le, A.-T., Lee, B.M.: NOMA in cooperative underlay cognitive radio networks under imperfect SIC. IEEE Access 8, 86180-86195 (2020)

24. He, B., Liu, A., Yang, N., Lau, V.K.N.: On the design of secure non-orthogonal multiple access systems. IEEE Journal on Selected Areas in Communications 35, 2196-2206 (2017) 
25. Wei, Z., Ng, D.W.K., Yuan, J., Wang, H.-M.: Optimal resource allocation for power-efficient MC-NOMA with imperfect channel state information. IEEE Transactions on Communications 65, 3944-3961 (2017)

26. Xingwang Li, Y.L.Z.D.A.N. Jingjing Li: Residual transceiver hardware impairments on cooperative NOMA networks. IEEE Transactions on Wireless Communications 19, 680-695 (2020)

27. Ding, Z., Liu, Y., Choi, J., Sun, Q., Elkashlan, M., Chih-Lin, I., Poor, H.V.: Application of non-orthogonal multiple access in LTE and 5 G networks. IEEE Communications Magazine 55, 185-191 (2017)

28. Ding, Z., Yang, Z., Fan, P., Poor, H.V.: On the performance of non-orthogonal multiple access in $5 \mathrm{G}$ systems with randomly deployed users. IEEE Signal Processing Letters 21, 1501-1505 (2014)

29. Ding, Z., Liu, Y., Choi, J., Sun, Q., Elkashlan, M., Chih-Lin, I., Poor, H.V.: Application of non-orthogonal multiple access in LTE and 5 G networks. IEEE Communications Magazine 55, 185-191 (2017)

30. He, B., Liu, A., Yang, N., Lau, V.K.N.: On the design of secure non-orthogonal multiple access systems. IEEE Journal on Selected Areas in Communications 35, 2196-2206 (2017)

31. Zheng, B., Wu, Q., Zhang, R.: Intelligent reflecting surface-assisted multiple access with user pairing: NOMA or OMA? IEEE Communications Letters 24, 753-757 (2020)

32. Ding, Z., Schober, R., Poor, H.V.: On the impact of phase shifting designs on irs-noma. IEEE Wireless Communications Letters 9, 1596-1600 (2020)

33. Zhang, Z., Zhang, C., Jiang, C., Jia, F., Ge, J., Gong, F.: Improving physical layer security for reconfigurable intelligent surface aided NOMA 6G networks. IEEE Transactions on Vehicular Technology 70, 4451-4463 (2021)

34. Hemanth, A., Umamaheswari, K., Pogaku, A.C., Do, D.-T., Lee, B.M.: Outage performance analysis of reconfigurable intelligent surfaces-aided NOMA under presence of hardware impairment. IEEE Access 8 , 212156-212165 (2020)

35. Chen, Q., Li, M., Yang, X., Alturki, R., Alshehri, M.D., Khan, F.: Impact of residual hardware impairment on the iot secrecy performance of ris-assisted noma networks. IEEE Access 9, 42583-42592 (2021)

36. Wu, D., Negi, R.: Effective capacity: a wireless link model for support of quality of service. IEEE Transactions on Wireless Communications 2, 630-643 (2003)

37. You, M., Sun, H., Jiang, J., Zhang, J.: Unified framework for the effective rate analysis of wireless communication systems over MISO fading channels. IEEE Transactions on Communications 65, 1775-1785 (2017)

38. Al-Badarneh, Y.H., Georghiades, C.N., Mejia, C.E.: On the effective rate of MISO/TAS systems in rayleigh fading. In: IEEE International Symposium on Information Theory (ISIT): 25-30 June 2017; Aachen, pp. 2328-2332 (2017)

39. Li, X., Li, J., Li, L., Jin, J., Zhang, J., Zhang, D.: Effective rate of MISO systems over $\kappa-\mu$ shadowed fading channels. IEEE Access 5, 10605-10611 (2017)

40. Hou, T., Liu, Y., Song, Z., Sun, X., Chen, Y., Hanzo, L.: Reconfigurable intelligent surface aided NOMA networks. IEEE Journal on Selected Areas in Communications 38, 2575-2588 (2020)

41. Liu, H., Ding, H., Xiang, L., Yuan, J., Zheng, L.: Outage and BER performance analysis of cascade channel in relay networks. In: The 9th International Conference on Future Networks and Communications (FNC-2014): 17-20 August 2014; Niagara Falls, pp. 23-30 (2014)

42. Gradshteyn, I.S., Ryzhik, I.M.: Table of integrals, series, and products, 7th edn., San Diego, CA, USA, p. 676 (2007) 
Figures

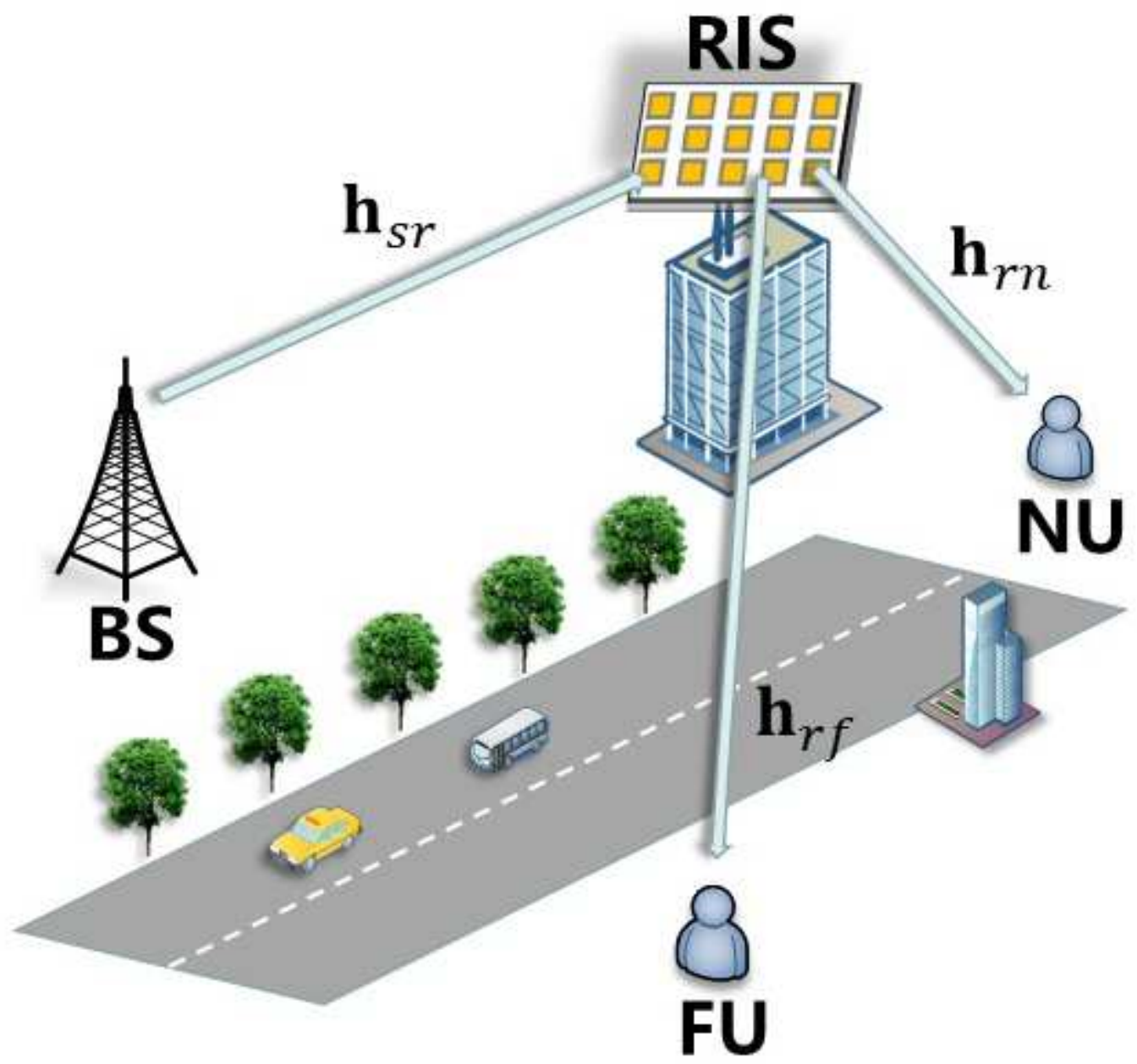

Figure 1

System Model 


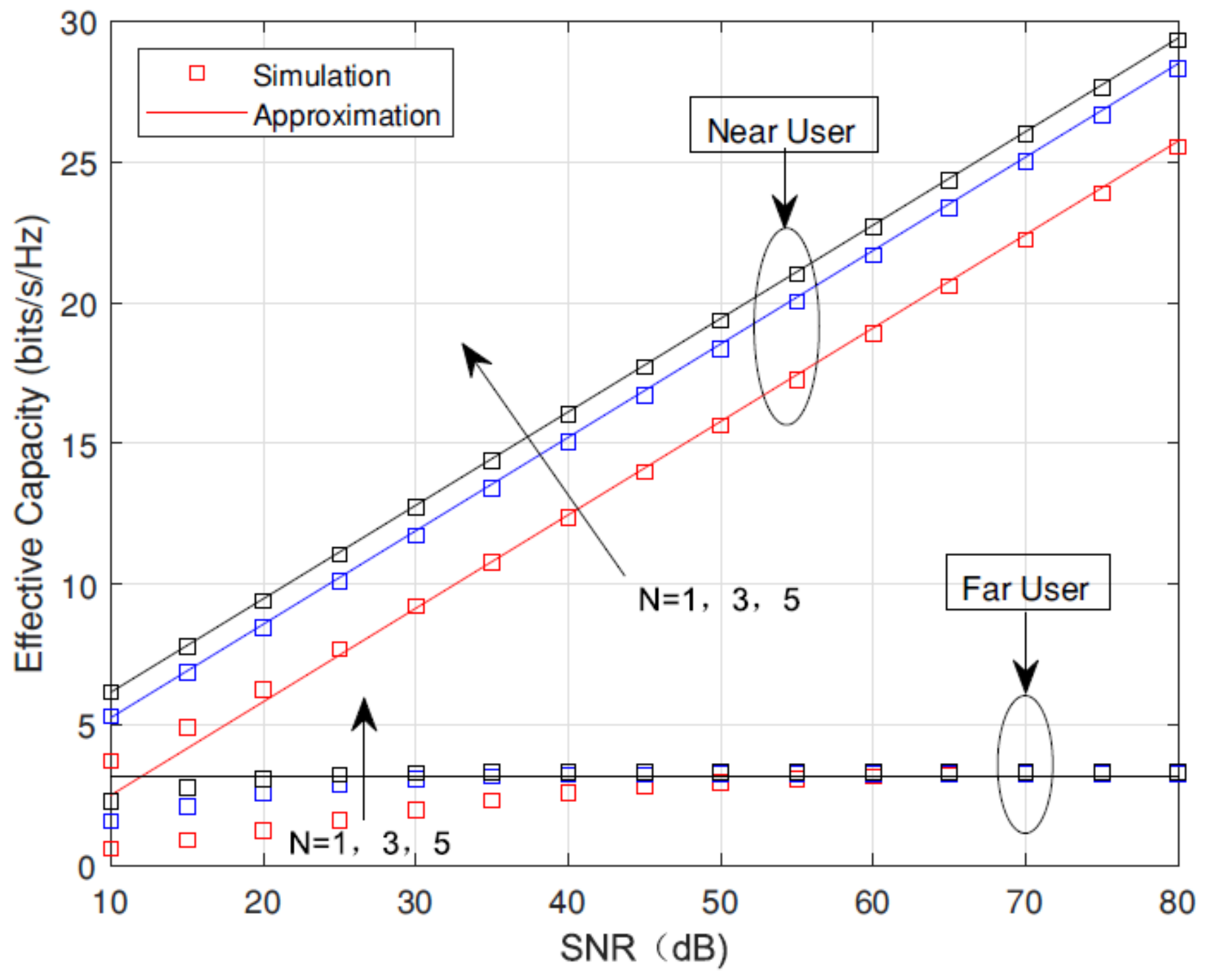

Figure 2

The EC relation with SNR on NU and FU 


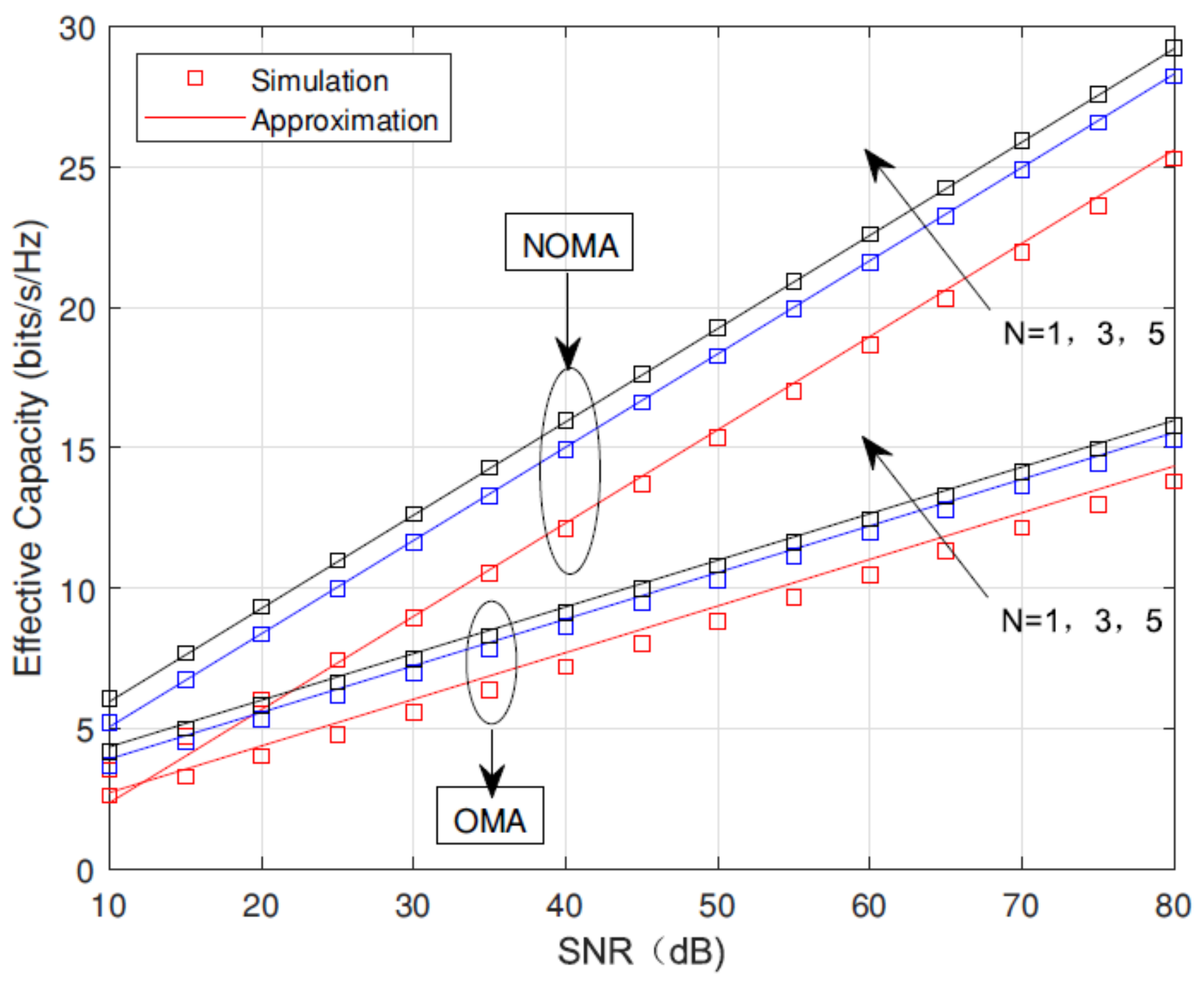

Figure 3

The EC relation with SNR in case of NOMA and OMA at NU 


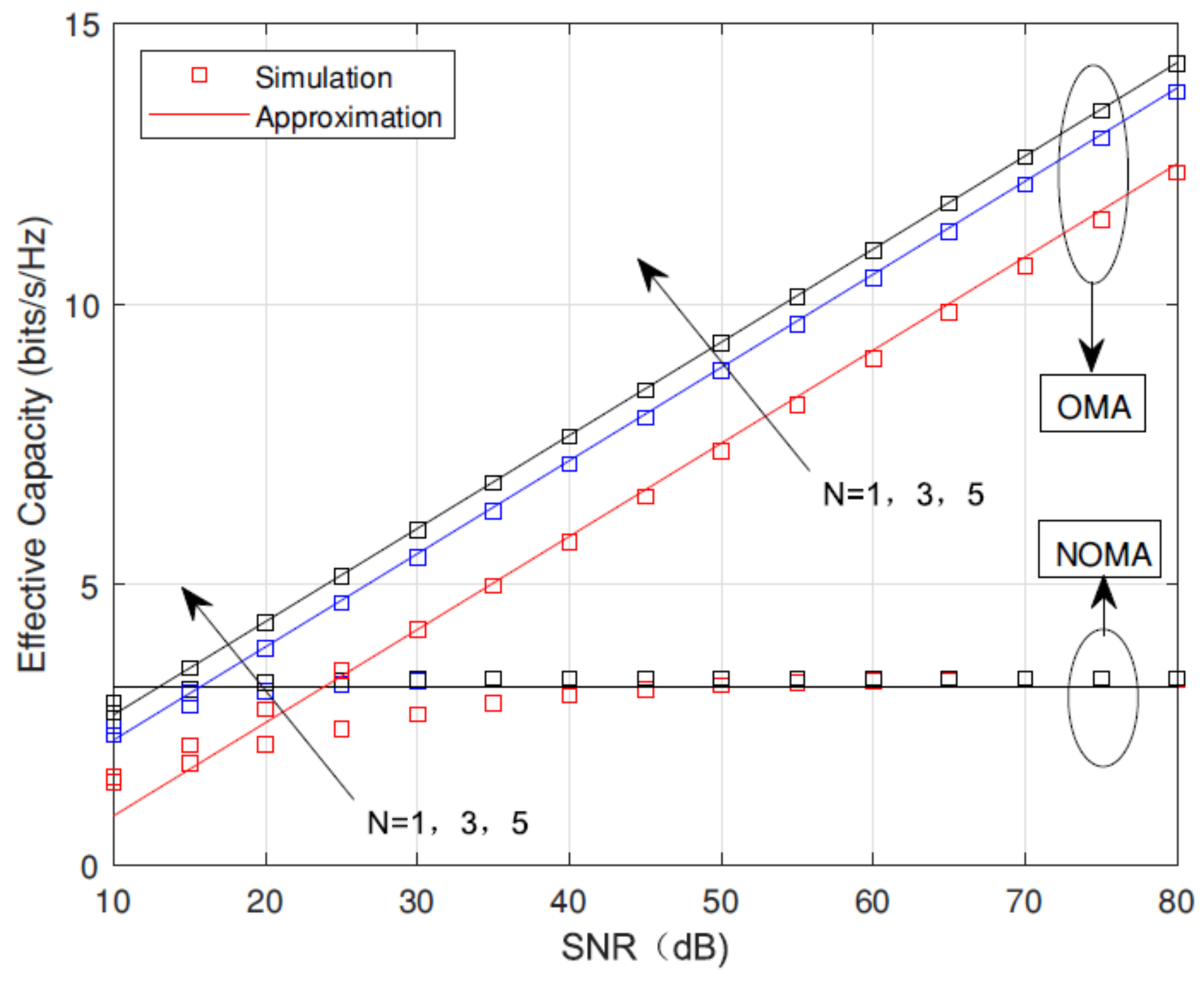

Figure 4

The EC relation with SNR in case of NOMA and OMA at NU 


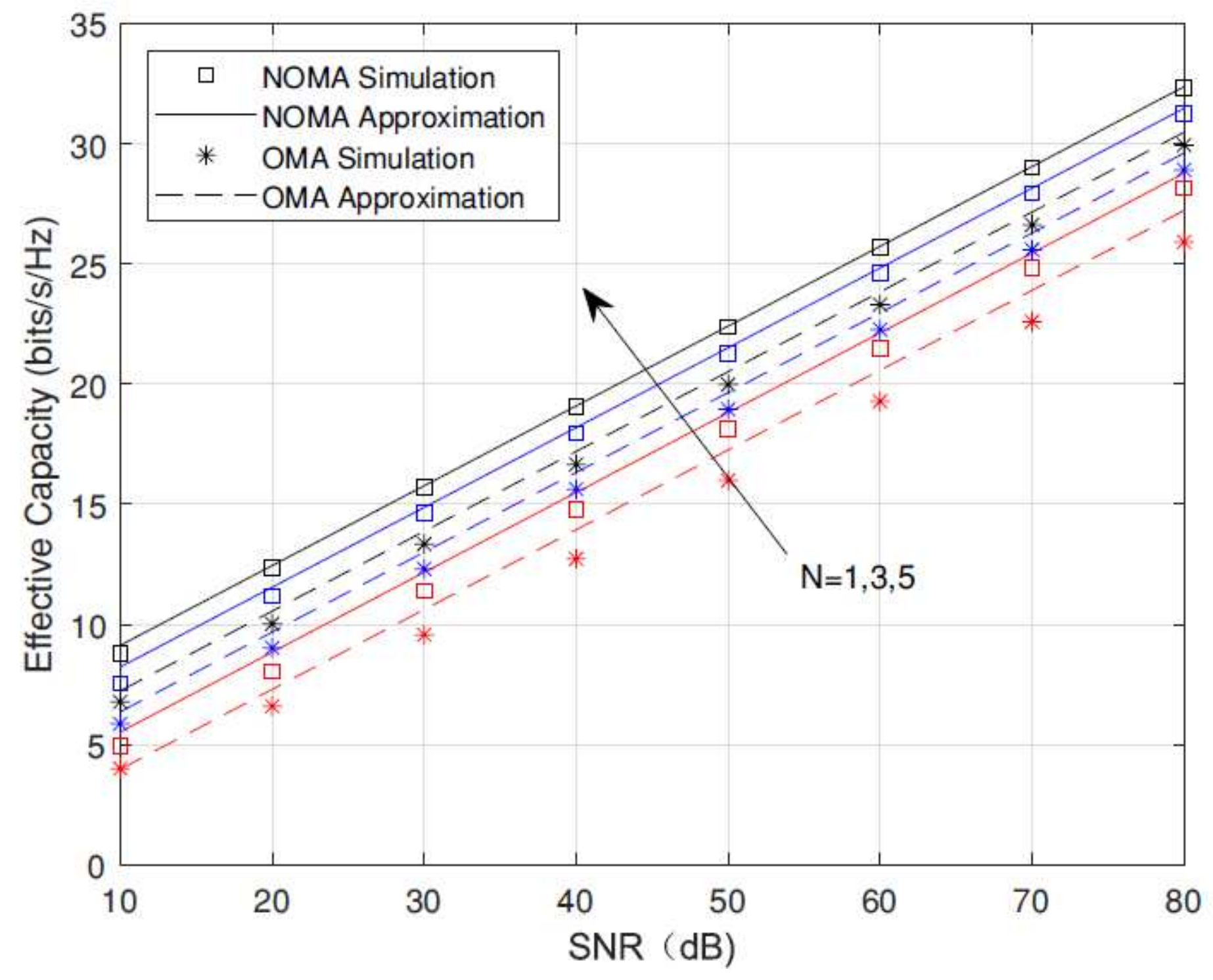

Figure 5

Comparison of the sum of EC between NOMA and OMA 


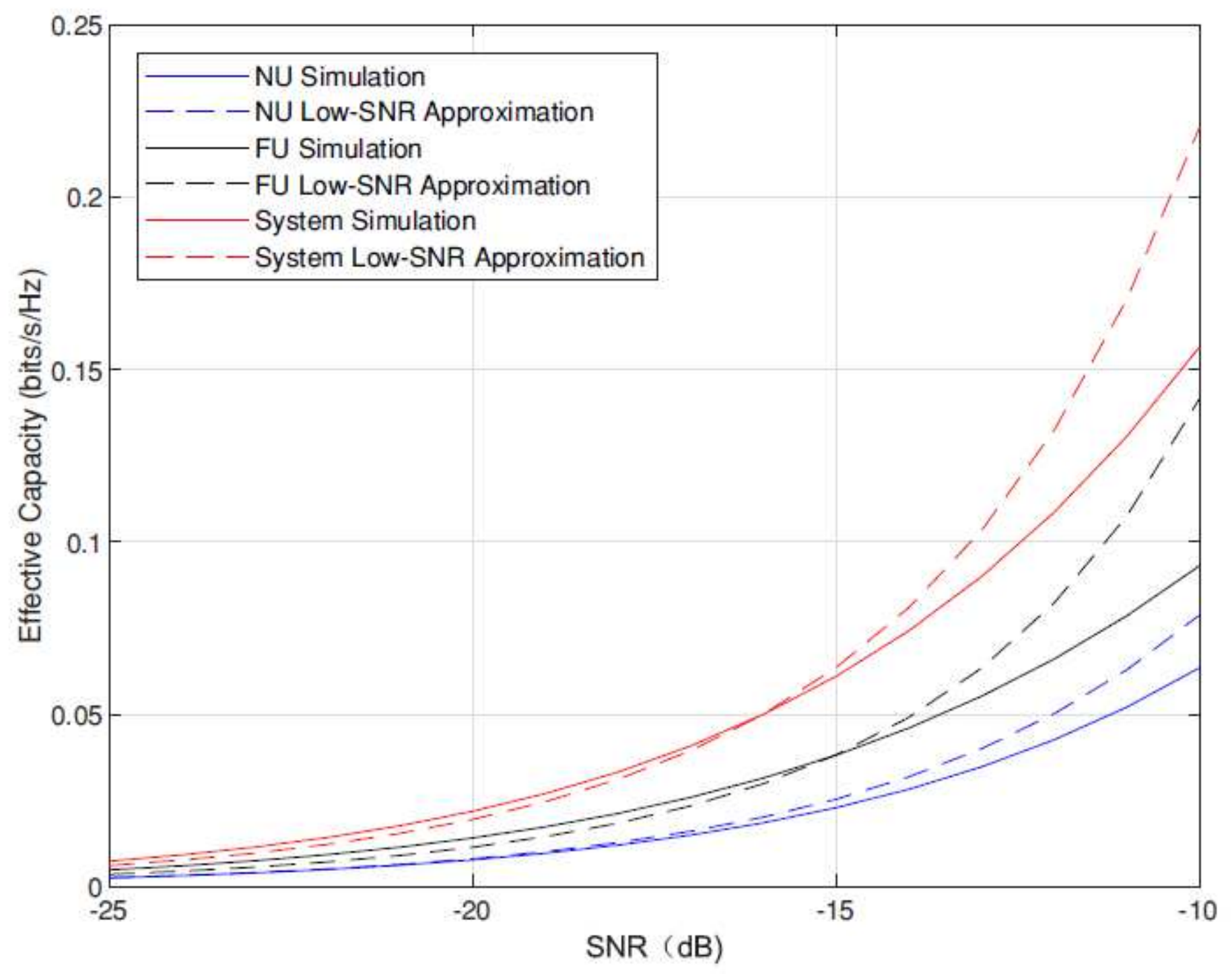

Figure 6

EC approximation with low-SNR with $\mathrm{N}=3$ 